\title{
Inelastic electron tunneling spectroscopy: A route to the identification of the tip-apex structure
}

\author{
Lucia Vitali, ${ }^{1}$ Svetlana D. Borisova, ${ }^{2}$ Galina G. Rusina, ${ }^{2}$ Evgueni V. Chulkov, ${ }^{3,4,5}$ and Klaus Kern ${ }^{1,6}$ \\ ${ }^{1}$ Max-Planck-Institut für Festkörperforschung, Heisenbergstr. 1, D-70569 Stuttgart, Germany \\ ${ }^{2}$ Institute of Strength Physics and Materials Science, pr. Academicheskii 2/1, 634021 Tomsk, Russia \\ ${ }^{3}$ Donostia International Physics Center (DIPC), Paseo de Manuel Lardizabal 4, 20018 San Sebastin/Donostia, Basque Country, Spain \\ ${ }^{4}$ Departamento de Física de Materiales, Facultad de Ciencias Qumicas, UPV/EHU, Apartado 1072, \\ 20080 San Sebastin/Donostia, Basque Country, Spain \\ ${ }^{5}$ Centro de Física de Materiales-CFM, CSIC-UPV/EHU, Apartado 1072, 20080 San Sebastin/Donostia, Basque Country, Spain \\ ${ }^{6}$ Institut de Physique de la Materière Condensée, Ecole Polytechnique Fédérale de Lausanne (EPFL), CH-1015 Lausanne, Switzerland
}

(Received 3 March 2010; published 21 April 2010)

\begin{abstract}
The vibrational spectrum of a tunneling junction on a clean $\mathrm{Cu}(111)$ surface has been characterized by vibrational density of states calculations and inelastic electron tunneling spectroscopy technique. We demonstrate that the achieved spectrum consists not only of vibrational modes excited by the tunneling electrons on the clean surface but also of modes characteristic of the structure of the tip apex. This allows to identify unequivocally the atomic structure of the tip, which is still the largest unknown parameter in a scanning tunneling microscope. This opens a new perspective in the interpretation of the measurements of vibrational modes with a scanning tunneling microscope. Additionally, it might have implications in the measurements of electron conductance through single atom or molecules contacted by the tip of scanning tunneling microscope.
\end{abstract}

DOI: 10.1103/PhysRevB.81.153409

PACS number(s): 68.37.Ef, 63.20.Pw, 68.35.Ja

The application of inelastic electron spectroscopy (IET) to characterize the lattice dynamics at the micrometer scale ${ }^{1-3}$ dates back to 1960s. Energy-loss excitations, assigned to phonons, have been clearly observed in the current vs voltage characteristics measured at a junction between two metals, as spear and anvil, pressed together to form a pointcontact junction. Specifically, in such a system, the measured inelastic signal results by a linear combination of the phonon spectra excited both at the spear and at the anvil. ${ }^{4}$ More recently, this spectroscopic technique has been extended successfully to scanning tunneling microscopy (IETS-inelastic electron tunneling spectroscopy) (Ref. 5) thus allowing characterization down to the atomic scale. Apart from the size of the probed area, the physical process behind the generation of the inelastic signal is the same in the two techniques: the lattice vibrations scatter the electrons passing through the junction and thus lead to characteristic energy-loss features. Despite this similarity, the origin of the inelastic spectra achieved with IETS-STM technique has been assigned exclusively to the vibrations excited at one electrode only. Indeed, so far only the lattice vibrations of surface supported nanostructures or of clean surfaces have been reported. ${ }^{5-13}$ Given the analogy between these two techniques, it is questionable if the inelastic spectrum achieved with IETS-STM truly account for the lattice vibration of one electrode only or if both surface and tip contribute to the scattering of the tunneling electrons.

The answer to this question goes beyond the fundamental level as it deals with the transport at nanoscale size junctions. Indeed, a scanning tunneling microscope can be applied to characterize the conductance through atomic or molecular junctions at distances ranging from tunneling to the pointcontact regime. ${ }^{14-18}$ These studies have demonstrated that inelastic contributions become increasingly more important as the dimensions of the solid are reduced down to the nanoscale level. These determine the electron transport and en- ergy dissipation at the junction, which critically depend by the atomistic structure at the junction.

Here, we demonstrate by combining scanning tunneling microscopy (STM) and spectroscopy (IETS-STM) with numerical simulations based on embedded atom method (EAM) that indeed the inelastic signal is constituted by lattice vibrations generated both at the surface and at the tip. As will be shown in the following, the spectrum experimentally recorded on a clean and topographically flat terrace using a copper terminated tip clearly indicates that features characteristic of the lattice dynamics of a clean $\mathrm{Cu}(111)$ surface ${ }^{19,20}$ are superimposed to phonon modes typical of small metal clusters. This copper surface is an ideal system to study the influence of the tip as the phonon spectrum of the surface is featureless in the low-energy range where the metal clusters show characteristic vibrational modes. These can therefore be distinguished and assigned to well-defined atomic structure of the tip apex, which in turn can be identified through the analysis of these modes.

The experiments were performed using a home-built scanning tunneling microscope operated at $6 \mathrm{~K}$ in ultrahigh vacuum (UHV) with a base pressure of $1 \times 10^{-11}$ mbar. The $\mathrm{Cu}(111)$ single crystal has been cleaned in UHV by cycles of $\mathrm{Ar}^{+}$-ion sputtering and annealing. The STM tip, chemically etched from tungsten wire, was treated in vacuo by electron field emission and soft indentation into the copper surface. This assured a spectroscopically featureless tip near the Fermi energy. Given this preparation, the tip was most likely covered by copper atoms derived from the substrate.

A representative inelastic electron tunneling spectrum obtained on a clean and topographically flat terrace on a $\mathrm{Cu}(111)$ surface is shown in Fig. 1(a). The arrows highlight three major vibrational features symmetrically located with respect to the Fermi level, as expected for lattice vibrations measured with IETS-STM. Indeed, in IETS-STM, the second-harmonic component of the modulated signal, which is proportional to the second derivative vs bias of the tunnel- 


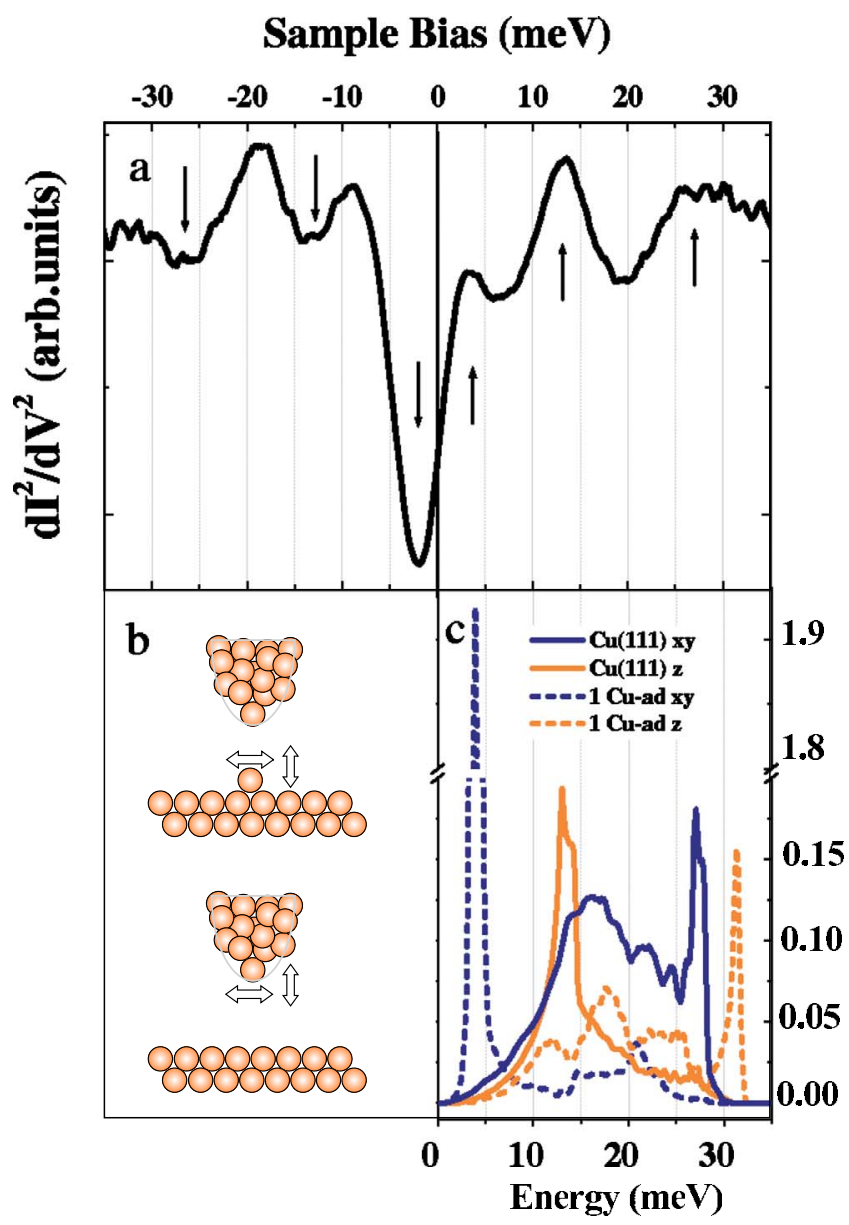

FIG. 1. (Color online) Vibrational spectra: (a) experimentally measured inelastic scanning tunneling spectrum on a clean $\mathrm{Cu}(111)$ surface. The inelastic electron tunneling spectrum was measured recording the tunneling current with a lock-in technique superimposing a sinusoidal signal with amplitude $\Delta V$ equal to $2.5 \mathrm{meV}$ (rms) to the applied sample bias. The arrows highlight three major vibrational features. (b) Sketch representing two different configurations which lead to identical inelastic tunneling electron spectroscopy spectra: (top) the $\mathrm{Cu}$ adatom is adsorbed on the surface or (below) or a single $\mathrm{Cu}$ adatom is located on the tip apex (below) and its inelastic electron tunneling signal adds to the one of the clean and flat $\mathrm{Cu}$ surface. (c) Theoretically calculated phonon spectra of a clean $\mathrm{Cu}(111)$ surface (solid line) and of a single $\mathrm{Cu}$ adatom on its surface (dotted), respectively. The in-plane $(x, y)$ and out-ofplane $(z)$ contributions are indicated in blue/black and orange/gray, respectively.

ing current, $d^{2} I / d V^{2}$, was recorded. In this way, the inelastic loss features in the conductance are transformed into peaks at the onset of the loss modes for positive sample bias and into minima for negative sample bias, respectively. These are centered at $3.4 \mathrm{eV}, 13.3 \mathrm{eV}$, and at about $27 \mathrm{meV}$, respectively. The last one, however, is broadened and extends to higher energies suggesting the contribution of several unresolved vibrational structures.

In order to increase the signal-to-noise ratio, the inelastic spectrum has been measured at reduced tip-sample distances by approaching the tip closer to the surface. The distance has been reduced stepwise from the tunneling conditions up to
$1.9 \AA$ where the spectra presented in this study have been measured. At each distance, the inelastic spectrum has been measured. Given that the energy of the phonon excitations was unchanged, we have concluded that the spectrum is not sensibly modified by tip-proximity effects. The tip-sample distance at the measured point has been estimated from point-contact experiments to be between 1.5 and $2 \AA$ from the surface.

Aiming for a clear identification of these structures, we first modeled the phonon spectrum of a fully relaxed clean $\mathrm{Cu}(111)$ surface $^{19}$ with EAM calculations. ${ }^{21,22}$ The phonon spectrum of this surface, projected onto the out-of-plane $z$ (orange/gray solid line) and onto the in-plane $x y$ (blue/black solid line) displacement of the copper atoms is presented in Fig. 1(c). These spectra show a rich phonon structure in perfect agreement with previously reported predictions and measurement achieved with other techniques (electron energy-loss spectroscopy ${ }^{23}$ and helium-atom scattering spectroscopy ${ }^{24}$ ). Two contributions dominate the phonon spectrum of this surface: an out-of-plane Rayleigh mode at $13.4 \mathrm{meV}$ and an in-plane mode at $27.9 \mathrm{meV}$. The energy position of these two modes is in very good agreement with two of the experimentally measured inelastic excitations.

It is, however, also evident that the phonon spectrum of the surface does not account for all the inelastic features experimentally measured. In particular, the vibration at lowest energy does not correspond to any of the predicted phonon modes of this copper surface.

As low-energy inelastic excitations are characteristic of small clusters of atoms, ${ }^{20}$ the vibrational spectrum of a single copper atom adsorbed onto the $\mathrm{Cu}(111)$ surface, as schematically drawn in the upper part of panel (b), has been calculated. The predicted vibration spectrum projected as in-plane and out-of-plane lattice displacement is shown in Fig. 1(c) (dotted lines). Two strong excitation modes corresponding to vibrations of the $\mathrm{Cu}$ adatom parallel $(3.9 \mathrm{meV})$ and perpendicular $(31.4 \mathrm{meV})$ to the surface can be seen. The latter can account for the asymmetric broadening of the energy-loss structure observed at about $27 \mathrm{meV}$ in the measured spectrum shown in Fig. 1(a). The predicted in-plane vibrational mode at low energy is in striking agreement with the experimental observation.

Considering that the experimentally measured spectrum has been achieved on a clean and flat surface, where no adatoms have been seen in the topographic image at the measured position, the very good agreement of the lowest-energy mode with the prediction for the in-plane vibration of a single adatom might be surprising. However, if we take into account that the vibration of the whole system can comprise the sum of the inelastic features of the surface and of the tip, a different interpretation is possible. We propose here that this mode reflects the inelastic excitation of a single copper adatom located at the tip apex, as sketched in the lower part of Fig. 1(b). The measured IETS-STM spectrum consists then of a superposition of the inelastic spectra of the clean $\mathrm{Cu}(111)$ surface and of that generated at the apex of the scanning tip. In fact, the two different topographic configurations sketched in Fig. 1(b), namely, the copper adatom located on the surface and the copper adatom located at the apex of the scanning tip generate the same vibrational spec- 
trum. The two configurations can only be distinguished through a topographic characterization of the measured position.

To further sustain our assignment that the observed inelastic features are a superposition of surface phonons and vibrational modes of the tip, we have measured inelastic spectra on the same surface with different tip apexes. These have been prepared by soft indentation into the clean surface (see Methods). In Fig. 2(a), we show a direct comparison of one such spectrum (blue/black line), with the original of Fig. 1(a) (orange/gray line).

At first glance, it is evident that the upper spectrum contains a larger number of vibrational features than the one obtained with a single-atom terminated tip previously discussed. The arrows, as a guide to the eyes, highlight the peaks positions. Knowing that the number of allowed lattice displacements increases with the increasing number of atoms forming a cluster, it can be expected that this spectrum is obtained by probing the vibrations of the $\mathrm{Cu}(111)$ surface with a non-monatomic tip apex.

In Fig. 2(b), we report the predicted vibrational spectrum of a system composed by a $\mathrm{Cu}(111)$ surface and a tip apex formed by a single or a 3-atoms-cluster termination (orange/ gray and blue/black line, respectively). The two predicted spectra differ therefore only in the contribution of the tip apex while the calculated surface contribution is the same in both cases. The predicted spectrum of the trimer terminated tip shows additional peaks in the low-energy region. These are in-plane vibrations corresponding to displacements of the entire trimer cluster and to its rotary vibration with respect to the center of mass (at $4.72 \mathrm{meV}$ and at $6.58 \mathrm{meV}$, respectively). The peak at $13.65 \mathrm{meV}$ contains two modes of the cluster (the antisymmetric vibration and the deformation vibration) and the surface Rayleigh mode previously discussed. At higher energy $(22.83 \mathrm{meV})$ also the shear vertical mode of the trimer can be seen.

The overall agreement between the theoretically predicted spectrum and the experimentally measured is excellent, allowing us to identify also in this case the structure of the tip apex. The only exception is the mode marked with the dotted arrow, which appears to be shifted by about $1.5 \mathrm{meV}$ toward lower energies with respect to the predictions.

This work clearly demonstrates that the IETS-STM spectrum measured on a metal surface contains contributions of two different origins: the surface modes and the vibration of the tip apex. This provides on one side a tool to distinguish through the tunneling electrons sharp and blunt tip apex and on the other side, it indicates that the phonon contributions of the tip play a significant role in the energy dissipation of the tunneling electrons. We believe that this has relevant consequences in the characterization of electron transport through

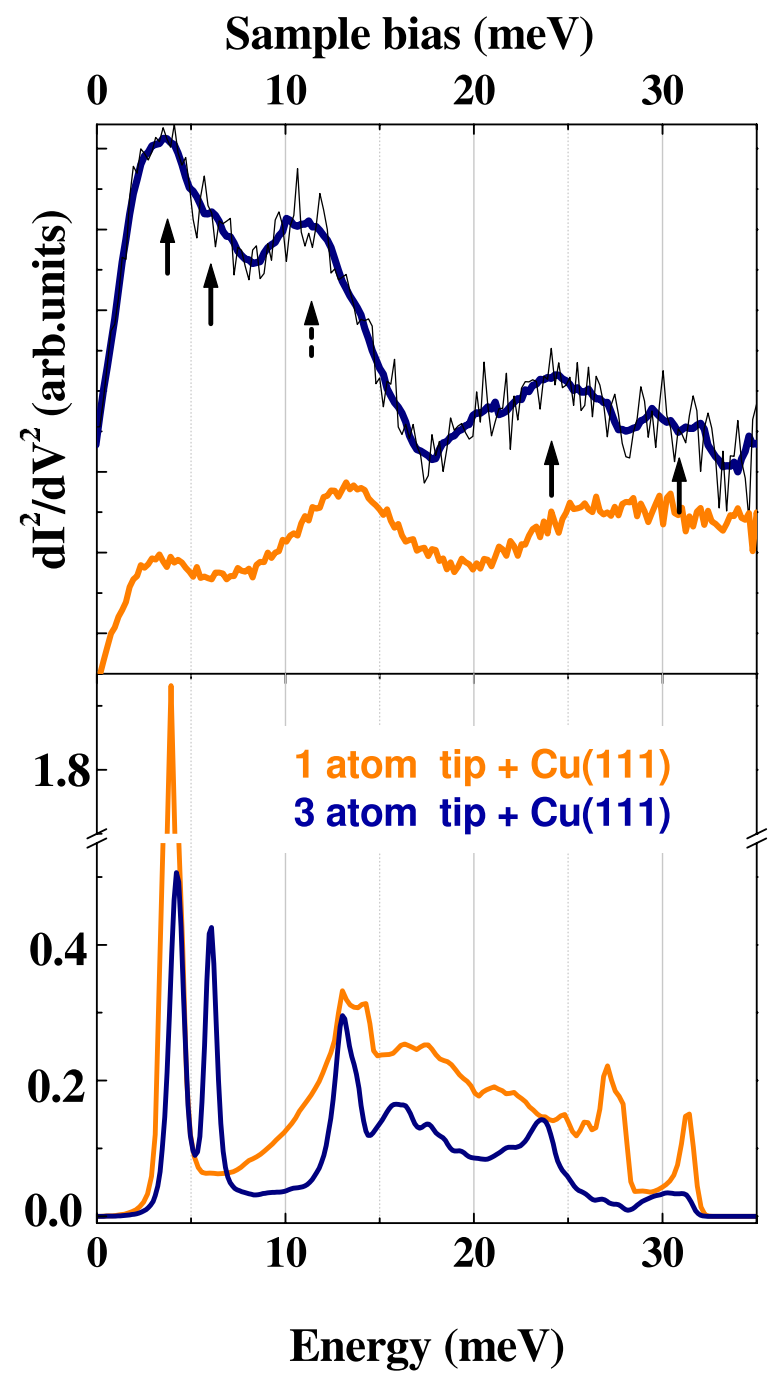

FIG. 2. (Color online) Tip configuration determining the vibrational spectra in a STM junction: (upper panel) experimentally measured inelastic tunneling spectra on a clean $\mathrm{Cu}(111)$ surface with two different tip terminations (orange/gray and blue/black lines). The increased number of peaks in the upper blue spectrum is indicative of a non-monatomic termination of the tip apex. (Lower panel) Theoretically predicted phonon spectra for a monoatomic (orange/gray line) and 3-atoms cluster on $\mathrm{Cu}(111)$ surface (blue/ black line). The orange/gray line is the sum of the predicted spectra for the surface and the single atom reported in Fig. 1(c).

nanoscale size junction, where the tip of the STM is used to contact adsorbed nanoscale structures. ${ }^{14-16}$ Indeed, several studies have demonstrated that not only do phonons influence the transport properties but also that they might lead to the instability of the junction. ${ }^{14,17,25}$ 
${ }^{1}$ Y. G. Naidyuk, and I. K. Yanson, Point Contact Spectroscopy, Spinger Series in Solid-State Science Vol. 145 (Springer-Verlag, New York, 2004).

${ }^{2}$ R. C. Jaklevic and J. Lambe, Phys. Rev. Lett. 17, 1139 (1966).

${ }^{3}$ E. L. Wolf, in Tunneling Spectroscopy, edited by P. K. Hansma (Plenum, New York, 1982).

${ }^{4}$ A. G. M. Jansen, F. M. Mueller, and P. Wyder, Phys. Rev. B 16, 1325 (1977).

${ }^{5}$ B. C. Stipe, M. A. Rezaei, and W. Ho, Science 280, 1732 (1998).

${ }^{6}$ A. J. Heinrich, C. P. Lutz, J. A. Gupta, and D. M. Eigler, Science 298, 1381 (2002).

${ }^{7}$ L. Vitali, M. A. Schneider, K. Kern, L. Wirtz, and A. Rubio, Phys. Rev. B 69, 121414(R) (2004).

${ }^{8}$ L. Vitali, M. Burghard, M. A. Schneider, L. Liu, S. Y. Wu, C. S. Jayanthi, and K. Kern, Phys. Rev. Lett. 93, 136103 (2004).

${ }^{9}$ L. Vitali, M. Burghard, P. Wahl, M. A. Schneider, and K. Kern, Phys. Rev. Lett. 96, 086804 (2006).

${ }^{10}$ H. Gawronski, M. Mehlhorn, and K. Morgenstern, Science 319, 930 (2008).

${ }^{11}$ N. Lorente and M. Persson, Phys. Rev. Lett. 85, 2997 (2000).

${ }^{12}$ J. I. Pascual, N. Lorente, Z. Song, H. Conrad, and H.-P. Rust, Nature (London) 423, 525 (2003).

${ }^{13}$ T. Komeda, Y. Kim, M. Kawai, B. N. J. Persson, and H. Ueba, Science 295, 2055 (2002).

${ }^{14}$ G. Schulze, K. J. Franke, and J. I. Pascual, New J. Phys. 10, 065005 (2008).

${ }^{15}$ L. Vitali, R. Ohmann, S. Stepanow, P. Gambardella, K. Tao, R. Huang, V. S. Stepanyuk, P. Bruno, and K. Kern, Phys. Rev. Lett. 101, 216802 (2008).

${ }^{16}$ N. Néel, J. Krőger, L. Limot, K. Palotas, W. A. Hofer, and R. Berndt, Phys. Rev. Lett. 98, 016801 (2007).
${ }^{17}$ Y. C. Chen, M. Zwolak, and M. Di Ventra, Nano Lett. 3, 1691 (2003).

${ }^{18}$ L. Vitali, A. Garcia-Lekue, T. Frederiksen, R. Ohmann, S. Sanchez-Portal, A. Arnau, and K. Kern, Nano Lett. 10, 657 (2010).

${ }^{19}$ S. D. Borisova, G. G. Rusina, S. V. Eremeev, G. Benedek, P. M. Echenique, I. Yu. Sklyadneva, and E. V. Chulkov, Phys. Rev. B 74, 165412 (2006).

${ }^{20}$ S. D. Borisova, S. V. Eremeev, G. G. Rusina, V. S. Stepanyuk, P. Bruno, and E. V. Chulkov, Phys. Rev. B 78, 075428 (2008).

${ }^{21}$ S. M. Foiles, M. I. Baskes, and M. S. Daw, Phys. Rev. B 33, 7983 (1986).

${ }^{22} \mathrm{The} \mathrm{Cu}$-metal surface clean and covered with $\mathrm{Cu}$ adatoms was modeled by a two-dimensional periodic slab consisting of 31 atomic layers of $\mathrm{Cu}(111)$ with supercells $(6 \times 6)$, large enough to exclude direct interactions between neighboring cells. The chosen thickness prevents also the interactions between the two opposite surfaces of the film. The $\mathrm{Cu}$ single adatoms and triangle clusters were arranged in a corresponding adsorption structure above both terminations of the slab. The calculations of vibrational spectra were carried out by the dynamical matrix method. Diagonalizing the matrix gives the frequencies and polarization vectors of vibrations. The local vibration densities of states were obtained by projecting these eigenmodes onto atoms of interest in a given $(x+y$ or $z)$ direction.

${ }^{23}$ Y. Chen, S. Y. Tong, J. S. Kim, L. L. Kesmodel, T. Rodach, K. P. Bohnen, and K. M. Ho, Phys. Rev. 44, 11394 (1991).

${ }^{24}$ G. Benedek and J. P. Toennies, Surf. Sci. 299-300, 587 (1994).

${ }^{25}$ M. Galperin, M. A. Ratner, and A. Nitzan, J. Phys.: Condens. Matter 19, 103201 (2007). 